\title{
From maximality to bias: Biased A-not-A questions in Mandarin Chinese* $^{*}$
}

\author{
Shumian Ye \\ Peking University
}

\begin{abstract}
This study aims to derive the epistemic bias in shi-bu-shi questions, a type of A-not-A question formed with the focus marker shi. I propose: (i) the focus marker shi presupposes that its prejacent is a possible complete answer to the current Question Under Discussion (QUD); (ii) accordingly, shi-bu-shi questions are presupposed to be part of the Focus-strategy of inquiry; (iii) the Focus-strategy of inquiry indicates the questioner's intention to close the current QUD, and to achieve this goal, the questioner should check the answer that she considers most likely to be true. By assuming such completeness-to-likelihood reasoning, a novel link between focus in polar questions and question bias is established. The ramifications of this proposal for related phenomena (e.g., bias in embedded questions, evidential bias) are then discussed.
\end{abstract}

Keywords: polar questions, focus, maximality presupposition, Question Under Discussion, strategies of inquiry, question bias

\section{Introduction}

Mandarin Chinese features two types of A-not-A questions. Whereas V-neg-V questions like (1) are used only in neutral contexts, shi-bu-shi questions like (2), formed with the focus marker shi, are used only in biased contexts where the questioner has a conjecture that the positive answer is true.

ni xihuan-bu-xihuan yuyongxue?

you like-not-like pragmatics

'Do you like pragmatics?'

(2) ni shi-bu-shi xihuan yuyongxue?

you SHI-not-SHI like pragmatics

'Is it pragmatics that you like?'

* For valuable discussions and comments, I thank Diti Bhadra, Gennaro Chierchia, Irene Heim, Ang Li, Maribel Romero, Roger Schwarzschild, Martin Stokhof, Tue Trinh, Danfeng Wu, and the audiences at Peking University, MIT, Harvard, LSA 2019, and SALT 30. I am also deeply indebted to Rui Guo, Manfred Krifka, and Mingming Liu for numerous helpful suggestions and encouragement.

C2020 Ye 
For instance, in (3), Zhangsan as the questioner lacks the knowledge of Lisi's research interests, and there is no clue in the context. To find out the addressee's research interests, the questioner can use V-neg-V questions but not shi-bu-shi questions. If the answer is yes, the addressee can respond by repeating the main verb of the question, but not by confirmative particles dui/meicuo/shide ('right'). ${ }^{1}$

(3) Neutral context: Zhangsan is interviewing Lisi, a student he knows nothing about. He is interested in Lisi's research interests.
$\mathrm{Q}:$ ni xihuan-bu-xihuan yuyongxue? you like-not-like pragmatics
A: \#dui/\#meicuo/\#shide/xihuan 'Do you like pragmatics?' \#right/like '\#Yes, you are right./Yes, I do.'

(4) illustrates a biased context in which public contextual evidence supports the positive answer. Since the questioner Zhangsan does not ignore the positive evidence (in the terminology of Sudo 2013), nor does he have prior beliefs, he will infer that the positive answer is more likely to be true. In contrast to (3), while Vneg-V questions are incompatible with the presence of positive evidence, shi-bu-shi questions are felicitous in such contexts, and can be answered by dui/meicuo/shide.

(4) Biased context: Zhangsan is curious about Lisi's research interests. In the library, he finds that Lisi is borrowing some books on pragmatics.
Q: ni shi-bu-shi xihuan yuyongxue?
you SHI-not-SHI like pragmatics
A: dui/meicuo/shide/\#xihuan right/\#like
'Is it pragmatics that you like?'
'Yes, you are right./\#Yes, I do.'

Positive evidence in the current context is not the only way to make the questioner biased. (5) exemplifies another type of biased context. Based on the prior hearsay, the questioner holds the private belief that the positive answer is more likely to be true. Again, shi-bu-shi questions, rather than V-neg-V questions, can be used in such contexts.

(5) Biased context: Zhangsan and Lisi are on the phone, and they are curious about each other's research interests. Zhangsan remembers someone telling him that Lisi liked pragmatics.

1 I take these confirmative particles as an additional diagnosis of question bias, given that they are used exclusively as responses to biased polar questions and assertions (see Guo 2000; Ye 2020a for details). I have developed an analysis of the semantics of these confirmative particles, which can be sketched as follows: (i) dui/meicuo/shide are remnants of the ellipsis of full clauses like $n i$ shuo de dui/meicuo/shide ('what you said is right'); (ii) they are anaphoric to a unique propositional discourse referent that calls for evaluation (cf. Krifka 2013); (iii) a propositional discourse referent calls for evaluation if the proposition is in the projected common grounds (Farkas \& Bruce 2010). 
From maximality to bias
Q: ni shi-bu-shi xihuan yuyongxue?
you SHI-not-SHI like pragmatics
A: dui/meicuo/shide/\#xihuan
right/\#like
'Is it pragmatics that you like?'
'Yes, you are right./\#Yes, I do.'

Both V-neg-V questions and shi-bu-shi questions are incompatible with the contextual evidence or the private belief that favors the negative answer. For instance, (1) and (2) are infelicitous in the following contexts:

(6) Biased context: Zhangsan is interested in Lisi's research interests. During the interview, he finds that Lisi has never read Paul Grice.

(7) Biased context: Zhangsan and Lisi are on the phone, and they are curious about each other's research interests. Zhangsan remembers someone telling him that Lisi did not like pragmatics.

To summarize, whereas V-neg-V questions can be characterized as anti-bias questions (Yuan \& Hara 2015), shi-bu-shi questions carry an epistemic bias towards the positive answer. ${ }^{2}$ Such positive epistemic bias is puzzling given that A-notA questions present two answer options $-\{\lambda w \cdot p(w), \lambda w \cdot \neg p(w)\}$ - equally and explicitly (as suggested by Krifka 2017).

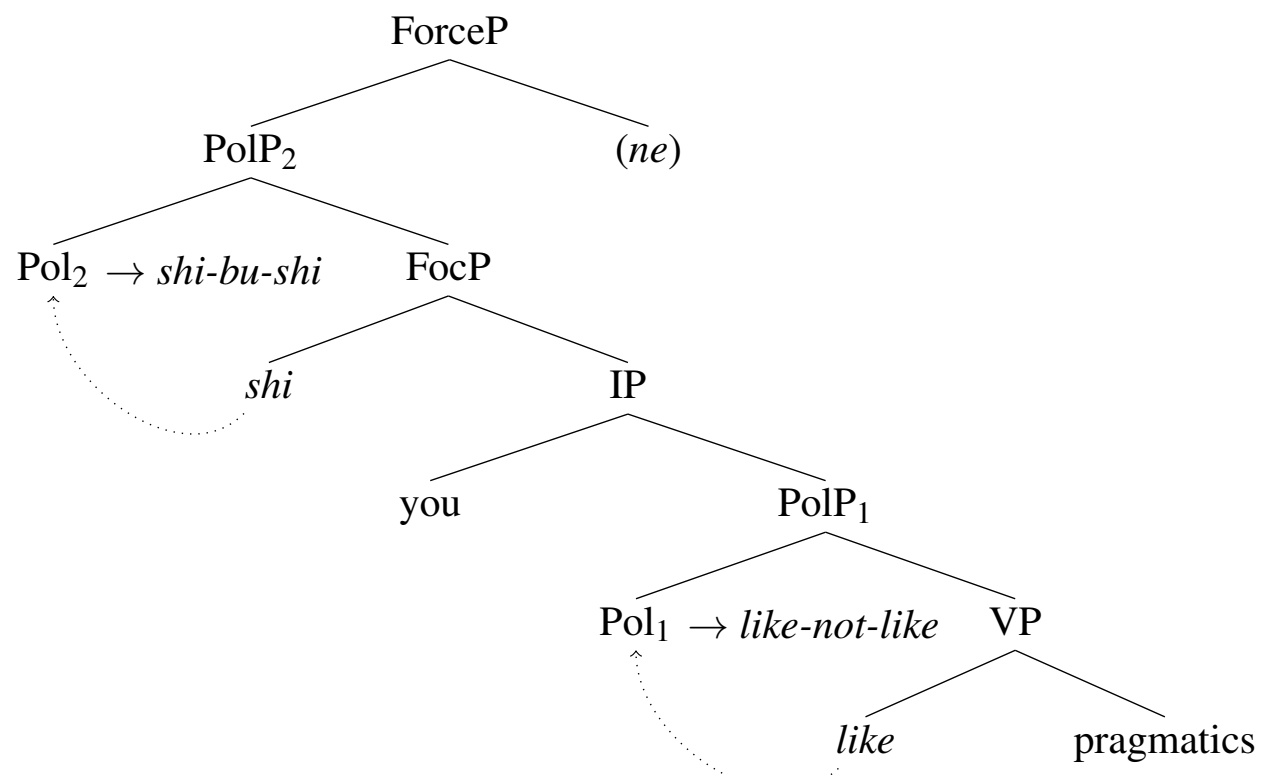

2 Note that the epistemic bias in shi-bu-shi questions is not a prior belief. As shown by (4) and (5), shi-bu-shi questions express the questioner's present conjecture, regardless of whether or not there exists supporting evidence in the current context. Sudo's (2013) division between evidential and epistemic bias does not appear to be significant for contexts like (4), in which there is no prior belief and the private belief is based on contextual evidence. 
Syntactically, the A-not-A form is generally assumed to be a morphological realization of the head of the Polarity Phrase (PolP), which functions as the Hamblin polar Q-operator taking a proposition and returning a set with the proposition and its negation (cf. Huang 1991; Schaffar \& Chen 2001). As sketched in the diagram above, shi-bu-shi questions differ from V-neg-V questions in the locus of the Polarity Phrase. Whereas V-neg-V is located inside the IP domain, shi-bu-shi, which is formed by reduplicating the focus marker shi and inserting the negator $b u$, is outside the IP domain (see Schaffar \& Chen 2001; Tsai \& Yang 2015; Ye 2020a for detailed evidence). It is uncontroversial that V-neg-V questions denote a size-two set. In view of the monopolar IP of shi-bu-shi questions, however, one may speculate that shi-bu-shi is lexicalized as some kind of speech act operator and shi-bu-shi questions denote a singleton set, which results in the epistemic bias. This possibility is unlikely given embedded A-not-A questions.

\section{Embedded by responsive predicates}

a. Zhangsan zhidao Lisi xihuan-bu-xihuan yuyongxue. Zhangsan know Lisi like-not-like pragmatics 'Zhangsan knows whether Lisi likes pragmatics.'

b. Zhangsan zhidao Lisi shi-bu-shi xihuan yuyongxue. Zhangsan know Lisi SHI-not-SHI like pragmatics 'Zhangsan knows whether it is pragmatics that Lisi likes.'

When embedded by certain responsive predicates (Lahiri 2002), the difference between V-neg-V questions and shi-bu-shi questions is insignificant, and the bias in shi-bu-shi questions seems concealed. ${ }^{3}$ For instance, (8a) and (8b) have the same truth conditions, that is, they are true just in case either Zhangsan knows that Lisi likes pragmatics or Zhangsan knows that Lisi doesn't like pragmatics. Neither the matrix subject Zhangsan nor the speaker considers it more likely that Lisi likes pragmatics. This suggests that the at-issue content of V-neg-V questions and shi$b u$-shi questions are the same, namely, the bipolar set $\{\lambda w \cdot p(w), \lambda w . \neg p(w)\}$.

This paper argues that shi-bu-shi questions differ from V-neg-V questions in not-at-issue content, and it is the presupposition of the focus marker shi that acts as the source of bias in shi-bu-shi questions. More specifically, I propose a threefold analysis to derive the positive epistemic bias in shi-bu-shi questions:

i. Maximality presupposition: the focus marker shi presupposes that its prejacent is a possible complete answer to the current Question Under Discussion (QUD).

3 I will return to this issue in Section 5.1. 
From maximality to bias

ii. Strategies of inquiry: according to the maximality presupposition, shi-bushi questions are presupposed to be part of the Focus-strategy of inquiry.

iii. Completeness-to-likelihood reasoning: the Focus-strategy of inquiry indicates the questioner's intention to close the current QUD, and to achieve this goal, the questioner should check the answer that she considers most likely to be true.

The remainder of this paper is structured as follows. Section 2 focuses on shideclaratives and shows that the focus marker shi carries the maximality presupposition proposed by Velleman, Beaver, Destruel, Bumford, Onea \& Coppock (2012). Section 3 points out that both focus and contrastive topic in polar questions indicate a strategy of inquiry, and shi-bu-shi questions are presupposed to be part of the Focus-strategy of inquiry. Section 4 proposes the completeness-to-likelihood reasoning, thereby forging a novel link between focus in polar questions and question bias. Section 5 discusses bias in embedded A-not-A questions and evidential bias.

\section{Maximality presupposition}

Before discussing the maximality presupposition, let me first clarify what is referred to as the focus marker shi and how it is distinguished from the copula shi and the stressed shi.

The focus marker shi can appear in sentence-initial or preverbal positions. The sentence-initial shi, as in (9a), only associates with the subject focus or the entire clause to its right, whereas the preverbal shi can freely associate with a focused constituent within its scope. The focused constituent is prosodically determined. ${ }^{4}$ For instance, (9b) has a narrow focus on the object, signaled by the main stress, and it can be used to answer the question 'which branches of linguistics does Lisi like?'. If nothing is particularly stressed, the whole constituent in the scope of shi, in this case the VP, is interpreted as a focus. The sentence then can address questions like 'what happens to Lisi?' and 'why is Lisi borrowing books on pragmatics?'.

Declaratives with the focus marker shi are exhaustive, shown by (9a) and (9b). The corresponding A-not-A questions, as in (9c), are the biased shi-bu-shi questions discussed in Section 1.

(9) Focus marker shi

a. shi LISI xihuan yuyongxue. (\#Zhangsan ye xihuan yuyongxue.) SHI Lisi like pragmatics Zhangsan also like pragmatics 'It is Lisi that likes pragmatics. (\#Zhangsan also likes pragmatics.)'

4 In this sense, shi simply marks the scope of focus.

5 See Paul \& Whitman (2008); Erlewine (2020) for more details about the focus association with shi. 
b. Lisi shi xihuan YUYONGXUE. (\#ta ye xihuan jufaxue.)

Lisi SHI like pragmatics he also like syntax

'It is pragmatics that Lisi likes. (\#He also likes syntax.)'

c. Lisi shi-bu-shi xihuan YUYONGXUE?

Lisi SHI-not-SHI like pragmatics

'Is it pragmatics that Lisi likes?'

POSITIVE BIAS

The copula shi is a verb taking a noun phrase as its complement. Copular sentences are non-exhaustive, as (10a) illustrates. A-not-A questions formed with the copula shi are neutral V-neg-V questions. For instance, (10b) is felicitous in a neutral context where the questioner is looking for pragmaticists and knows nothing about Lisi.

It seems that (10b) can also be used in a biased context where the questioner suspects that Lisi is a pragmaticist. I argue that such biased A-not-A questions, however, are in fact formed with the focus marker shi, and the copula shi is deleted in PF by haplology, as (10c) illustrates. This is demonstrated by (10d) - when adverbs intervene, the copula shi does not immediately follow the focus marker shi, and thus must be pronounced.

\section{(10) Copula shi}

a. Lisi shi yige yuyongxuejia. ta ye shi yige jufaxuejia.

Lisi COP one pragmaticist he also COP one syntactician

'Lisi is a pragmaticist. He is also a syntactician.'

b. Lisi shi-bu-shi yige yuyongxuejia?

Lisi COP-not-COP one pragmaticist

'Is Lisi a pragmaticist?'

NEUTRAL

c. Lisi shi-bu-shi shi yige yuyongxuejia?

Lisi SHI-not-SHI COP one pragmaticist

'Lisi is a pragmaticist, right?'

POSITIVE BIAS

\section{d. Lisi shi-bu-shi zhilye *(shi) yige yuyongxuejia?}

Lisi SHI-not-SHI only/also COP one pragmaticist

'Lisi is only/also a pragmaticist, right?'

POSITIVE BIAS

When the focus marker shi is stressed, the declaratives turn non-exhaustive and receive a VERUM-like reading; compare (11a) with (9b). Stressed shi-bu-shi questions express a complex bias: the questioner holds the prior belief that the positive answer is true, but there exists contradictory evidence in the current context, so the questioner double-checks the prior belief. In addition, stressed shi-bu-shi questions exhibit the cornering effect, i.e., they force the addressee to give an answer (cf. Biezma 2009; Biezma \& Rawlins 2012, 2017). 
From maximality to bias

\section{(11) Stressed shi}

a. Lisi SHI xihuan yuyongxue. ta ye xihuan jufaxue.

Lisi SHI like pragmatics he also like syntax

'Lisi DOES like pragmatics. He also likes syntax.'

b. Lisi (daodi) SHI-bu-SHI xihuan yuyongxue?

Lisi at.all SHI-not-SHI like pragmatics

'Is it really pragmatics that Lisi likes or not?'

COMPLEX BIAS

It is the stress itself, not the stressed $s h$, that gives rise to the VERUM-like reading of declaratives and the complex bias with the cornering effect in A-not-A questions. (12) shows the same pattern for stressed verbs. Stressed V-neg-V questions also have the cornering effect, yet the bias is slightly different: both the positive and the negative answer are supported by diverse contextual evidence and/or beliefs, and therefore the questioner asks the addressee to resolve the conversational crisis (Farkas \& Bruce 2010).

\section{(12) Stressed verbs}

a. Lisi XIHUAN yuyongxue. ta ye xihuan jufaxue. Lisi like pragmatics he also like syntax 'Lisi DOES like pragmatics. He also likes syntax.'

b. Lisi (daodi) XIHUAN-bu-XIHUAN yuyongxue?

Lisi at.all like-not-like pragmatics

'Does Lisi really like pragmatics or not?'

COMPLEX BIAS

(11) seems to support Romero \& Han's (2004) VERUM approach to question bias, as stressed shi-bu-shi questions behave analogously to high negative polar questions in English with respect to bias. It is plausible to assume that the stress introduces a VERUM-like operator. But to account for the absence of exhaustivity in (11a), the VERUM-like operator must trivialize the presupposition of the focus marker shi in some way. I leave the full analysis of (11) and (12) for a later occasion.

In a nutshell, the focus marker shi differs from the copula shi and the stressed shi in exhaustivity and question bias, as outlined in the table below.

\begin{tabular}{l|l|l}
\hline & Exhaustivity in declaratives & Bias in A-not-A questions \\
\hline Focus marker shi & exhaustive & positive epistemic bias \\
Copula $s h i$ & non-exhaustive & neutral \\
Stressed $s h i$ & non-exhaustive & complex bias \& cornering effect \\
\hline
\end{tabular}

Now let us concentrate on the focus marker shi. As shown by (13) and (14), $s h i$-declaratives give rise to three types of inference, akin to it-clefts in English. 
(13) Lisi shi xihuan YUYONGXUE.

Lisi SHI like pragmatics

'It is pragmatics that Lisi likes.'

$\rightsquigarrow$ There exists a branch of linguistics that Lisi likes.

EXISTENTIAL

$\rightsquigarrow$ Lisi does not like other branches.

EXHAUSTIVE

$\rightsquigarrow$ Someone thought that Lisi might like other branches.

CONTRASTIVE

(14) Lisi bu-shi xihuan YUYONGXUE.

Lisi not-SHI like pragmatics

'It is not pragmatics that Lisi likes.'

$\rightsquigarrow$ There exists a branch of linguistics that Lisi likes.

$\rightsquigarrow$ Lisi likes other branches.

EXISTENTIAL

$\rightsquigarrow$ Someone thought that Lisi might like pragmatics.

NON-EXHAUSTIVE

CONTRASTIVE

The existential inference is presuppositional, for it is backgrounded and can be inherited by negative sentences (and polar questions). The exhaustive inference does not survive under negation, but as we have seen in (9), it is not easily cancellable. The contrastive inference is backgrounded, and varies with the polarity of the sentence. ${ }^{6}$ To provide a unified analysis of these inferences, I adopt the maximality presupposition by Velleman et al. (2012) and propose that the focus marker shi presupposes that its prejacent is a possible strongest/complete answers to the current QUD. ${ }^{7}$

a. $\operatorname{MAX}_{S}(p)=\lambda w . \forall q \in C Q_{S}\left[\left(q>_{S} p\right) \rightarrow \neg q(w)\right]$

b. $\llbracket \mathrm{SHI} \rrbracket^{S}=\lambda p \cdot \lambda w: \operatorname{MAX}_{S}(p)(w) \cdot p(w)$

c. $\llbracket \mathrm{BU}-\mathrm{SHI} \rrbracket^{S}=\neg \llbracket \mathrm{SHI} \rrbracket^{S}=\lambda p \cdot \lambda w: \operatorname{MAX}_{S}(p)(w) \cdot \neg p(w)$

In words: $\operatorname{SHI}(p)$ and $\mathrm{BU}-\operatorname{SHI}(p)$ presuppose that among the possible answers to the current QUD in the context $S\left(C Q_{S}\right)$, no true answer is strictly stronger than $p$.

6 For the contrastive inference in it-clefts, see Destruel \& Velleman (2014); Destruel, Beaver \& Coppock (2018).

7 I do not adopt the full cleft semantics by Velleman et al. (2012). They propose another focussensitive operator $\operatorname{MIN}_{S}(p)$ for the at-issue content of $i$-clefts, which requires that there be a true answer at least as strong as the prejacent $p$ :

(i) $\operatorname{MIN}_{S}(p)=\lambda w \cdot \exists q \in C Q_{S}\left[q(w) \wedge\left(q \geq_{S} p\right)\right]$

(ii) $\operatorname{CLEFT}_{S}=\lambda p \cdot \lambda w: \operatorname{MAX}_{S}(p)(w) \cdot \operatorname{MIN}_{S}(p)(w)$

For their discussions on it-clefts, replacing $\operatorname{MIN}_{S}(p)$ with $p$ causes no change. As for shideclaratives, the 'at least' inference is not independently attested (cf. Coppock \& Beaver 2011, 2014). Furthermore, as evidenced by (8), shi-bu-shi questions and V-neg-V questions share the same at-issue content, so the focus marker shi only contributes to the not-at-issue content.

Erlewine (2020) proposes that the semantics of shi is CLEFT. As I have argued, however, the MIN component is not empirically motivated for shi, and makes incorrect predictions for (8). 


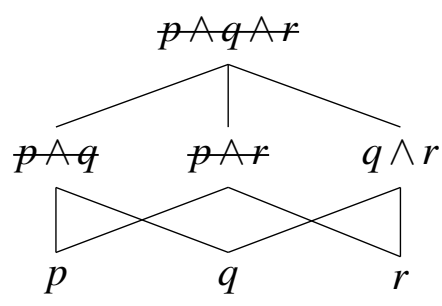

Figure 1 Stronger answers to the current QUD eliminated by $\operatorname{MAX}_{S}(p)$

The current QUD is the salient question in the context, which can be modeled as a set of Rooth-Hamblin alternatives (Roberts 1996/2012; Beaver \& Clark 2008). Focus in assertions indicates the QUD, as assertions are presupposed to be congruent to the QUD, that is, the focus alternative set of an assertion is required to be identical to the QUD set (Roberts 1996/2012). Assuming that the alternatives in the QUD set, i.e., the possible answers to the QUD, are partially ordered by entailment, the maximality presupposition $\operatorname{MAX}_{S}(p)$ restricts the QUD set by eliminating possible answers that both entail and are distinct from $p$, as illustrated in Figure 1.

Consider the shi-declaratives in (13) and (14), repeated as (16). The object focus activates alternatives such as 'Lisi likes syntax' and 'Lisi likes semantics', thereby indicating the QUD 'which branches of linguistics does Lisi like?' represented by (16c). Since the maximality presupposition of shi makes reference to the QUD, (16a) and (16b) can be used only if the speaker and the addressee accept the question in (16c) and their common conversational goal is to find the true answer (Roberts 1996/2012). The existential presupposition of shi-declaratives, then, comes from the presupposed QUD which requires both the speaker and the addressee to believe the existence of a true answer. ${ }^{8}$
a. Lisi shi likes pragmatics $F$.
b. Lisi $b u$-shi likes pragmatics $F$.
c. $C Q_{S}=\{$ like $($ prag $)$, like $($ syn $)$, like $($ sem $)$, like $($ syn $\oplus$ sem $)$,

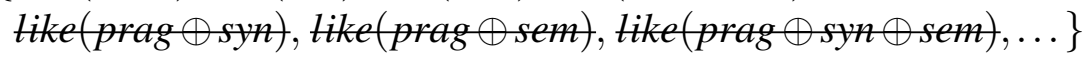

8 The existential presupposition of shi-declaratives is stronger than that of regular $w h$-questions. Dayal (2016: 51-52) suggests that the distinction between the soft existential presupposition of regular $w h$-questions and the hard existential presupposition of clefted wh-questions lies in whether the addressee is presupposed to believe the existence of a true answer:

(i) Who left? (Presupposition: the speaker believes that someone left.)

(ii) Who was it that left? (Presupposition: both the speaker and the addressee believe that someone left.) 
The maximality presupposition of shi eliminates stronger alternatives in the QUD set, which leads to the polarity asymmetry with respect to exhaustive inference. Both (16a) and (16b) presuppose the QUD set in (16c), where stronger alternatives like 'Lisi likes pragmatics and syntax' are ruled out. (16a) asserts that Lisi likes pragmatics; if Lisi also liked another branch, e.g., syntax, the stronger alternative 'Lisi likes pragmatics and syntax' would be true, contradicting the presupposed QUD set in (16c). Hence, (16a) gives rise to the exhaustive inference that Lisi does not like other branches. (16b) asserts that it is false that Lisi likes pragmatics; nevertheless, in the QUD set there exist other alternatives that have no entailment relationship with the prejacent, from which one can draw the nonexhaustive inference that Lisi likes other branches.

Basically, the prejacent of shi is required to be a possible complete answer to the QUD, which is mutually exclusive with other possible complete answers. The contrastive inference follows from the mutual exclusivity. If the prejacent is asserted to be true, other possible complete answers are false. Thus, (16a) can be used to exclude all other possibilities considered by the (potential) addressee. If the prejacent is asserted to be false as in (16b), the sentence excludes the possibility that the prejacent is the true complete answer. This possibility was typically on the table in the previous context, as required by the 'discourse presupposition' of negative sentences (Givón 1978).

\section{Strategies of inquiry}

I analyze shi in shi-bu-shi questions as the same focus marker, so the maximality presupposition projects out of shi-bu-shi questions. As illustrated in (17), shi-bu-shi questions differ from $\mathrm{V}$-neg- $\mathrm{V}$ questions only in the maximality presupposition.

$$
\begin{aligned}
& \text { V-neg-V questions: }\{\lambda w \cdot p(w), \lambda w \cdot \neg p(w)\} \\
& \text { shi-bu-shi questions: }\left\{\lambda w: \operatorname{MAX}_{S}(p)(w) \cdot p(w), \lambda w: \operatorname{MAX}_{S}(p)(w) . \neg p(w)\right\}
\end{aligned}
$$

Given the maximality presupposition, shi-bu-shi questions are used to check the truth of possible complete answers to the current QUD, which indicates a strategy of inquiry, namely, a set of questions partially ordered by entailment (Roberts 1996/2012). Büring (2003) proposes that whereas focus in assertions generates a set of propositions that cannot indicate a strategy, contrastive topic in assertions generates a set of questions and thus indicates a strategy. However, polar questions do not appear to receive full consideration. In the following, I will argue that both focus and contrastive topic in polar questions indicate a strategy of inquiry, and shi-bu-shi questions are presupposed to be part of the Focus-strategy of inquiry.

Suppose the current QUD in the context is "which branches of linguistics does Lisi like?'. Its possible complete answers, as in (18a), are exhaustive and mutually 
exclusive, and therefore they are disjunctive alternatives. By contrast, the set of possible partial answers in (18b) only includes atoms that are non-exhaustive and can be conjoined.

a. Complete answers: $\{$ Lisi only likes pragmatics, Lisi only likes syntax, Lisi only likes pragmatics and syntax,$\ldots\}$

b. Partial answers: $\{$ Lisi likes pragmatics, Lisi likes syntax,$\ldots\}$

Correspondingly, there are two ways to find the true complete answer to the QUD. The questioner can use a polar question as a subquestion to check the truth of a possible complete answer. If the answer to the polar question is yes, the QUD will be closed; if not, the questioner should move to the next subquestion.

The questioner can also use polar questions to check the truth of possible partial answers. The QUD will not be closed until the questioner has gone through all the subquestions. As a consequence, no matter whether the answer to a non-terminal subquestion is yes or no, the questioner should move to the next subquestion.

Polar questions in the first strategy are accompanied by focus, while polar questions with contrastive topic are generally used in the second strategy. Thus, I dub these two strategies the F(ocus)-strategy of inquiry and the C(ontrastive)T(opic)strategy of inquiry, respectively.

\begin{tabular}{|c|c|}
\hline$Q U D$ & Which branches of linguistics does Lisi like? \\
\hline \multicolumn{2}{|c|}{ F-strategy of inquiry (Checking the truth of possible complete answers) } \\
\hline Subquestions & Is it pragmatics ${ }_{F}$ that Lisi likes? \\
\hline \multirow[t]{2}{*}{ Answers } & Yes - close the QUD \\
\hline & No-move to the next subquestion \\
\hline \multicolumn{2}{|c|}{ CT-strategy of inquiry (Checking the truth of possible partial answers) } \\
\hline Subquestions & (How about pragmatics $C T$ ?) Does Lisi like pragmatics ${ }_{C T}$ ? \\
\hline \multirow[t]{2}{*}{ Answers } & Yes - move to the next subquestion \\
\hline & No-move to the next subquestion \\
\hline
\end{tabular}

I assume, following Kamali \& Krifka (2020), that focus and contrastive topic trigger disjunctive and conjunctive alternatives, respectively. Given the congruence requirement, disjunctive and conjunctive alternatives to assertions correspond to possible complete and partial answers to the QUD, and accordingly, disjunctive and conjunctive alternatives to polar questions are subquestions of the QUD, checking the truth of possible complete and partial answers (see also Ye 2020b). Thereby, both focus and contrastive topic in polar questions indicate a strategy of inquiry.

The maximality presupposition of shi requires shi-bu-shi questions to be part of the F-strategy. Consider for example (5), repeated here as (19a). Since the 
answer to the shi-bu-shi question is yes, the questioner gets the complete answer that Lisi only likes pragmatics and the current QUD is closed. It is infelicitous for the questioner to follow up with an inquiry about another alternative; such antiadditivity is analogous to exhaustivity in shi-declaratives.

\section{(19) Focus in assertions and polar questions}

a. Q: You shi-bu-shi like pragmatics ${ }_{F}$ ?

A: Yes, you are right.

\#Q: Do you like syntax?

b. Lisi shi likes pragmatics $F$. (\#He also likes syntax.)

By contrast, V-neg-V questions can be used to form the CT-strategy. ${ }^{9}$ Consider the V-neg-V question in (20a), repeated from (3). After receiving the positive answer, the questioner only learns that Lisi likes pragmatics, and therefore can further inquire about other branches. In this sense, contrastive topics exhibit anti-exhaustivity in polar questions as in declaratives.

\section{(20) Contrastive topic in assertions and polar questions}

a. Q: You like-not-like pragmatics ${ }_{C T}$ ?
A: Yes, I do.
Q: Do you like syntax?

b. Lisi likes pragmatics $C T$. He also likes $\operatorname{syntax}_{C T}$.

For the cases discussed above, (anti-)exhaustivity and (anti-)additivity follow from completeness of answerhood. Since contrastive topics represent partial answers to the QUD and shi requires its prejacent to be a complete answer, shi cannot associate with a contrastive topic. This prediction is borne out by (21). ${ }^{10}$

Zhangsan likes all branches of linguistics; ...

a. *Lisi ne, shi TA xihuan yuyongxue.

Lisi CT SHI he like pragmatics

Intended: 'As for $\operatorname{Lisi}_{C T}$, it is he ${ }_{F}$ that likes pragmatics.'

b. *Lisi ne, shi-bu-shi TA xihuan yuyongxue?

Lisi CT SHI-not-SHI he like pragmatics

Intended: 'As for $\operatorname{Lisi}_{C T}$, is it he ${ }_{F}$ that likes pragmatics?'

9 V-neg-V questions never occur in the F-strategy, which can be attributed to the pragmatic principle Maximize Presupposition! (Heim 1991). Roughly speaking, since V-neg-V questions and shi-bu-shi questions have the same at-issue content and differ only in the maximality presupposition, the use of V-neg-V questions implicates that the maximality presupposition does not hold, that is, V-neg-V questions are not used to check possible complete answers.

10 Thanks to Mitcho Erlewine for raising this issue. Here I take the particle ne as a contrastive topic marker, following Constant (2014). See Liu (2017) for a similar test on Mandarin focus particles. 
From maximality to bias

The focus marker shi can co-occur with contrastive topics when there is no association between them. For instance, (21) will be grammatical if shi associates with the object focus, as in (22).

Zhangsan likes all branches of linguistics; ...

a. Lisi ne, ta shi xihuan YUYONGXUE.

Lisi CT he SHI like pragmatics

'As for $\operatorname{Lisi}_{C T}$, it is pragmatics ${ }_{F}$ that he likes.'

b. Lisi ne, ta shi-bu-shi xihuan YUYONGXUE?

Lisi CT he SHI-not-SHI like pragmatics

'As for $\operatorname{Lisi}_{C T}$, is it pragmatics $F$ that he likes?'

In this case, 'it is pragmatics that Lisi likes' is a possible complete answer to the question 'as for Lisi, which branches of linguistics does he like?', which itself inquires about a partial answer to the big question 'which branches of linguistics do they like?'. The discourse tree is as follows (cf. Büring 2003):

Which branches of linguistics do they like?

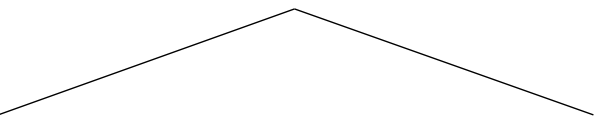

As for Lisi $C T$, which branches does he like? As for Zhangsan ${ }_{C T}, \ldots$

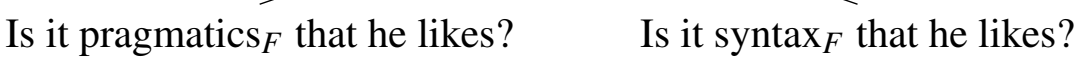

\section{From completeness to likelihood}

In the previous section, we have seen that the F-strategy differs from the CT-strategy in the completeness of answerhood and the informativity with respect to the QUD. In the F-strategy, (i) polar questions are used as subquestions to check the truth of possible complete answers; (ii) positive answers to the subquestions can close the QUD. In the CT-strategy, (i) polar questions are used as subquestions to check the truth of possible partial answers; (ii) the QUD will not be closed until the questioner has gone through all the possible partial answers.

The hallmark of the F-strategy is that once the possible complete answer is true, the QUD will be closed, which conforms to the notion of 'inquiry terminating' in Velleman et al. (2012). Since the F-strategy is a faster strategy for closing the QUD, I assume that the F-strategy indicates the questioner's intention to close the QUD, and further propose the completeness-to-likelihood reasoning for the F-strategy. 


\section{(24) Completeness-to-likelihood reasoning}

In the F-strategy, the possible complete answer checked by the topmost polar question is the one that the questioner considers most likely to be true.

a. The questioner will not adopt the F-strategy unless she intends to close the QUD.

b. If the questioner adopts the F-strategy and intends to close the QUD, she expects a positive answer (i.e., $p>_{\text {expected }} \neg p$ ), given that only positive answers to the subquestions can close the QUD.

c. If the questioner expects a positive answer, she should check the possible complete answer that she considers most likely to be true (i.e., $\forall q \in$ $\left.C Q_{S}\left[(q \neq p) \rightarrow\left(p>_{\text {likely }} q\right)\right]\right)$, rather than a random one. ${ }^{11}$

The positive epistemic bias in shi-bu-shi questions follows directly from the completeness-to-likelihood reasoning. Since shi-bu-shi questions are presupposed to be part of the F-strategy, the questioner expects a positive answer to close the QUD, and the possible complete answer that she is checking is the one that she considers most likely to be true.

Note that the completeness-to-likelihood reasoning hinges on the assumption in (24a). (24b) follows from (24a) and the 'inquiry terminating' property of the Fstrategy. (24c) follows from (24b) and the mutual exclusivity of possible complete answers. Given that the main conversational goal is to close the QUD (Roberts 1996/2012), it seems natural to assume that strategies of inquiry in general indicate the questioner's intention to close the QUD. For the F-strategy, there are two possible contexts.

First, in a context where both the F-strategy and the CT-strategy are available, the questioner will not choose the F-strategy unless she intends to close the QUD in a faster way. If the questioner cannot or does not want to close the QUD in a faster way, she will choose the CT-strategy. If the questioner has no intention of closing the QUD at all, she can express her ignorance ('I have no idea') or indifference ('I don't care'), and no strategy of inquiry will be adopted.

Second, when the F-strategy is the only strategy available in the context, the speaker has two choices: try to close the QUD via the F-strategy, or refuse to take an active part in the conversational game. For instance, in guessing games, a guesser is often forced to adopt the F-strategy. A participant can remain silent or admit her ignorance, but once she provides a possible complete answer, she is taken as an active guesser, and the possible complete answer is taken as the one that she considers most likely to be true.

11 Given the mutual exclusivity of possible complete answers, if the questioner expects one possible complete answer to be true, she expects others to be false. Hence, no matter how the questioner assesses the likelihood of each possible complete answer, there must be a unique winner. 
From maximality to bias

As for the CT-strategy, since the questioner must go through all the possible partial answers to close the QUD, the possible partial answers need not be ranked, and the questioner in principle can check the possible partial answers randomly. In other words, I predict that polar questions with contrastive topic are typically used in neutral contexts. This prediction is supported by (25).

(25) Scenario: The speaker is organizing a party and she is in charge of supplying all the non-alcoholic beverages for teetotalers. The speaker is going through a list of people that are invited. She has no previous belief or expectation about their drinking habits.

A: Jane and Mary do not drink.

S: OK. What about John? Does he not drink (either)?

While Romero \& Han (2004) provide this example to show that low negative polar questions are not necessarily biased, it is worth pointing out here that the negative polar question in (25) is used as a subquestion in the CT-strategy. To wit, the QUD in this context is 'who do not drink?' and 'John does not drink' is a possible partial answer.

I conclude that the F-strategy differs from the CT-strategy in the likelihood relations between subquestions. In the F-strategy, (iii) the subquestions are ranked in descending order of likelihood. In the CT-strategy, (iii) the subquestions need not be ranked.

By assuming the completeness-to-likelihood reasoning, a novel link between focus in polar questions and question bias is established. It can further capture the context-dependence of bias in unmarked positive polar questions. For instance, bias in $m a$-questions, the run-of-the-mill polar questions in Mandarin Chinese, is largely context-dependent (Guo 2000). Consider the Q\&A pairs below. The ma-question is biased in (26), but neutral in (27), as evidenced by the $d u i$-answer diagnosis (see footnote 1). This contrast can be attributed to the presupposition of the preceding wh-QUD. In (26), the singular which-question carries a uniqueness presupposition, and the ma-question proffers a possible complete answer to the which-question, thereby forming the F-strategy. By contrast, in (27), the plural which-question asks for a plural answer, and the $m a$-question checks a possible partial answer. As a subquestion in the CT-strategy, the ma-question is predicted to be neutral.

$\mathrm{Q}:$ ni jian-le na-yige ren? ni jian-le Lisi ma?

you meet-ASP which-one person you meet-ASP Lisi Q

'Which person did you meet? Did you meet Lisi ${ }_{F}$ ?'

\section{A: jian-leldui}

meet-ASP/right

'Yes, I did./Yes, you are right.' 
$\mathrm{Q}:$ ni jian-le na-liangge ren? ni jian-le Lisi ma?

you meet-ASP which-two person you meet-ASP Lisi Q

'Which two people did you meet? Did you meet Lisi $_{C T}$ ?'

\section{A: jian-le/\#dui}

meet-ASP/\#right

'Yes, I did./\#Yes, you are right.'

The completeness-to-likelihood reasoning predicts that polar questions in the F-strategy convey a positive epistemic bias. However, $m a$-questions with the focus marker shi can occur in neutral contexts, which seems to be a counterexample. ${ }^{12}$ For instance, in the following neutral context, ma-questions with the focus marker shi and V-neg-V questions are felicitous, in contrast to shi-bu-shi questions.

(28) Neutral context: The teacher said that she lost one student's homework. Zhangsan does not know whose homework has been lost, but he is very concerned about his own homework.

a. shi wo-de zuoye diu-le ma?

SHI I-POSS homework lost-ASP Q

'Was my homework lost?'

b. wo-de zuoye diu-mei-diu?

I-POSS homework lost-not-lost

'Was my homework lost?'

c. \#shi-bu-shi wo-de zuoye diu-le?

SHI-not-SHI I-POSS homework lost-ASP

'\#Was it my homework that was lost?'

Note that the questioner Zhangsan is not concerned about whose homework was lost, but rather about whether his homework was lost. If the answer to the polar question is no, Zhangsan's worry will be removed and he will stop inquiring. Put differently, in (28), no wh-QUD is presupposed and no strategy of inquiry is involved. Hence, (28a) is not a genuine counterexample to the completeness-tolikelihood reasoning.

Nevertheless, it is not obvious why the focus marker shi in ma-questions does not necessarily presuppose the F-strategy. I would like to suggest that the contribution of the question particle $m a$ is to add its prejacent to the top of the QUD stack. The wh-QUD presupposed by shi can be either added to the QUD stack or suppressed by ma. If the shi ...ma question is itself the current QUD, the maximality presupposition of $s h i$ is trivially satisfied, yet no strategy of inquiry is indicated.

12 Thanks to Jad Wehbe and Yu' an Yang for bringing this to my attention. 
From maximality to bias

\section{Discussion}

\subsection{Bias in embedded questions}

As mentioned in the introduction, the bias in shi-bu-shi questions seems concealed when embedded by certain responsive predicates. For instance, the embedded shibu-shi question in (29a), just like the embedded V-neg-V question in (29b), does not give rise to any inference with respect to bias. Neither the matrix subject Zhangsan nor the speaker considers it more likely that Lisi likes pragmatics.

\section{(29) Embedded by responsive predicates}

a. Zhangsan zhidao Lisi shi-bu-shi xihuan yuyongxue. Zhangsan know Lisi SHI-not-SHI like pragmatics 'Zhangsan knows whether it is pragmatics that Lisi likes.' $\Varangle \rightarrow$ Zhangsan conjectures that Lisi may like pragmatics.

b. Zhangsan zhidao Lisi xihuan-bu-xihuan yuyongxue. Zhangsan know Lisi like-not-like pragmatics 'Zhangsan knows whether Lisi likes pragmatics.' $\not \rightarrow$ Zhangsan conjectures that Lisi may like pragmatics.

By contrast, when embedded by some rogative predicates, shi-bu-shi questions convey a positive epistemic bias, yet the attitude holder is shifted to the matrix subject. For instance, (30a) carries the inference that the matrix subject is biased towards the positive answer to the embedded question, whereas (30b) does not.

\section{(30) Embedded by rogative predicates}

a.tamen zai yilun Lisi shi-bu-shi xihuan yuyongxue. they at talk.about Lisi SHI-not-SHI like pragmatics 'They are talking about whether it is pragmatics that Lisi likes.' $\rightsquigarrow$ They conjecture that Lisi may like pragmatics.

\section{b. tamen zai yilun Lisi xihuan-bu-xihuan yuyongxue.} they at talk.about Lisi like-not-like pragmatics 'They are talking about whether Lisi likes pragmatics.' $\not \rightarrow$ They conjecture that Lisi may like pragmatics.

Intuitively, the contrast between (29a) and (30a) can be attributed to the role of the matrix subject. The subjects of responsive predicates canonically take the role of answerer, whereas the subjects of some rogative predicates take the role of questioner. According to the completeness-to-likelihood reasoning, the bias in shi$b u$-shi questions stems from the questioner's intention and expectation, while the answerer's epistemic state is irrelevant. Thus, embedded bias can be observed with certain rogative predicates. I leave the technical implementation for future work. 


\subsection{Evidential bias}

As demonstrated by (4) and (5) in the introductory section, shi-bu-shi questions are not sensitive to the distinction between evidential and epistemic bias (see also footnote 2). Consider now the following biased context adapted from Sudo (2013):

(31) Biased context: Zhangsan and Lisi work in a windowless room. When Lisi enters the room, Zhangsan notices that his jacket is wet.

Q: shi-bu-shi xiayu-le?

SHI-not-SHI rain-ASP

'Is it raining?'

In the classical view, (31) illustrates a context with positive evidential bias, and the question bias is directly connected to the contextual evidence, i.e., the compelling contextual evidence supports or contextually entails the positive answer (Büring \& Gunlogson 2000; Gunlogson 2003; Reese 2007; Sudo 2013; a.o.). Given my proposal for shi-bu-shi questions, there seems to be an alternative way of deriving question bias from the contextual evidence:

i. The contextual evidence $q$ can raise an implicit (potential) question $Q$ (e.g., 'why $q$ ?'), in the sense of Onea (2016).

ii. To ask a polar question ' $p$ ?' in the context with the evidence $q$, is typically to check the possible complete answer $p$ to the implicit question $Q$.

iii. By the completeness-to-likelihood reasoning, ' $p$ ?' is biased towards $p$.

Take (31) for illustration. When noticing Lisi's wet jacket, Zhangsan may wonder why. In this sense, the contextual evidence raises an implicit question 'why is Lisi's jacket wet?'. By asking 'is it raining?', Zhangsan wants to check with Lisi whether the possible complete answer 'it is raining' is true. In accordance with the completeness-to-likelihood reasoning, the questioner expects the positive answer.

Note that in the second step, there is no guarantee that the polar question will be used to check the possible complete answer. To remedy this, I assume that implicit (potential) questions like why-questions by default carry a uniqueness presupposition. Further investigation is needed to confirm this assumption.

While the QUD view of contextual evidence blurs the distinction between evidential and epistemic bias in certain cases, it captures the QUD requirement of shi-bu-shi questions and helps us understand why such questions are insensitive to the EVI-EPI distinction. Moreover, it provides another perspective on how contextual evidence and question bias are related. The contextual evidence may not contextually entail or imply the positive answer directly, but rather introduces a set of alternatives including the positive answer. 
From maximality to bias

\section{References}

Beaver, David I. \& Brady Z. Clark. 2008. Sense and sensitivity: How focus determines meaning. Wiley-Blackwell.

Biezma, María. 2009. Alternative vs Polar Questions: The cornering effect. In Ed Cormany, Satoshi Ito \& David Lutz (eds.), Proceedings of Semantics and Linguistic Theory 19, 37-54. Ithaca, NY: CLC. doi:10.3765/salt.v19i0.2519.

Biezma, María \& Kyle Rawlins. 2012. Responding to alternative and polar questions. Linguistics and Philosophy 35(5). 361-406. doi:10.1007/s10988-0129123-z.

Biezma, María \& Kyle Rawlins. 2017. Or what? Semantics and Pragmatics 10(16). 1-44. doi:10.3765/sp.10.16.

Büring, Daniel. 2003. On D-Trees, beans, and B-Accents. Linguistics and Philosophy 26(5). 511-545. doi:10.1023/A:1025887707652.

Büring, Daniel \& Christine Gunlogson. 2000. Aren't positive and negative polar questions the same? Manuscript, UCSC. https://semanticsarchive.net/Archive/ mYwOGNhO/polar_questions.pdf.

Constant, Noah. 2014. Contrastive topic: Meanings and realizations: University of Massachusetts Amherst PhD dissertation.

Coppock, Elizabeth \& David Beaver. 2011. Sole sisters. In Neil Ashton, Anca Chereches \& David Lutz (eds.), Proceedings of Semantics and Linguistic Theory 21, 197-217. Ithaca, NY: CLC. doi:10.3765/salt.v21i0.2615.

Coppock, Elizabeth \& David I. Beaver. 2014. Principles of the exclusive muddle. Journal of Semantics 31(3). 371-432. doi:10.1093/jos/fft007.

Dayal, Veneeta. 2016. Questions. New York: Oxford University Press.

Destruel, Emilie, David Beaver \& Elizabeth Coppock. 2018. Clefts: Quite the contrary! In Robert Truswell, Chris Cummins, Caroline Heycock, Brian Rabern \& Hannah Rohde (eds.), Proceedings of Sinn und Bedeutung 21, vol. 1, 335346. University of Edinburgh.

Destruel, Emilie \& Leah Velleman. 2014. Refining contrast: Empirical evidence from the English it-cleft. In Christopher Piñón (ed.), Empirical Issues in Syntax and Semantics 10, 197-214. Université Paris 7.

Erlewine, Michael Yoshitaka. 2020. Mandarin shì clefts and the syntax of discourse congruence. Manuscript. https://ling.auf.net/lingbuzz/005176.

Farkas, Donka F. \& Kim B. Bruce. 2010. On reacting to assertions and polar questions. Journal of Semantics 27(1). 81-118. doi:10.1093/jos/ffp010.

Givón, Talmy. 1978. Negation in language: Pragmatics, function, ontology. In Peter Cole (ed.), Syntax and semantics (Volume 9): Pragmatics, 69-112. New York: Academic Press.

Gunlogson, Christine. 2003. True to form: Rising and falling declaratives as ques- 
tions in English. New York/London: Routledge.

Guo, Rui. 2000. Credibility degree of ma-questions and corresponding answers. Shijie Hanyu Jiaoxue [Chinese Teaching in the World] 2(13-23). doi:10.13724/j.cnki.ctiw.2000.02.002.

Heim, Irene. 1991. Artikel und definitheit. In Arnim von Stechow \& Dieter Wunderlich (eds.), Semantik: Ein internationales handbuch der zeitgenössischen forschung, 487-535. Berlin/Boston: De Gruyter.

Huang, C.-T. James. 1991. Modularity and Chinese A-not-A questions. In Carol Georgopoulos \& Roberta Ishihara (eds.), Interdisciplinary approaches to language: Essays in honor of S.-Y. Kuroda, 305-332. Dordrecht: Springer Netherlands.

Kamali, Beste \& Manfred Krifka. 2020. Focus and contrastive topic in questions and answers, with particular reference to Turkish. Theoretical Linguistics 46(12). 1-71. doi:10.1515/tl-2020-0001.

Krifka, Manfred. 2013. Response particles as propositional anaphors. In Todd Snider (ed.), Proceedings Semantics and Linguistic Theory 23, 1-18. Ithaca, NY: CLC. doi:10.3765/salt.v23i0.2676.

Krifka, Manfred. 2017. Negated polarity questions as denegations of assertions. In Chungmin Lee, Ferenc Kiefer \& Manfred Krifka (eds.), Contrastiveness in information structure, alternatives and scalar implicatures, 359-398. Cham: Springer International Publishing Switzerland.

Lahiri, Utpal. 2002. Questions and answers in embedded contexts. New York: Oxford University Press.

Liu, Mingming. 2017. Varieties of alternatives: Mandarin focus particles. Linguistics and Philosophy 40(1). 61-95. doi:10.1007/s10988-016-9199-y.

Onea, Edgar. 2016. Potential questions at the semantics-pragmatics interface. Leiden/Boston: Brill.

Paul, Waltraud \& John Whitman. 2008. Shi ... de focus clefts in Mandarin Chinese. The Linguistic Review 25(3-4). 413-451. doi:10.1515/TLIR.2008.012.

Reese, Brian Jon. 2007. Bias in questions: The University of Texas at Austin PhD dissertation.

Roberts, Craige. 1996/2012. Information structure: Towards an integrated formal theory of pragmatics. Semantics and Pragmatics 5(6). 1-69. doi:10.3765/sp.5.6.

Romero, Maribel \& Chung-Hye Han. $2004 . \quad$ On negative yes/no questions. Linguistics and Philosophy 27(5). 609-658. doi:10.1023/B:LING.0000033850.15705.94.

Schaffar, Wolfram \& Lansun Chen. 2001. Yes-no questions in Mandarin and the theory of focus. Linguistics 39(5). 837-870. doi:10.1515/ling.2001.036.

Sudo, Yasutada. 2013. Biased polar questions in English and Japanese. In Daniel 
From maximality to bias

Gutzmann \& Hans-Martin Gärtner (eds.), Beyond expressives: Explorations in use-conditional meaning, 275-295. Leiden: Brill.

Tsai, Wei-Tien Dylan \& Ching-Yu Helen Yang. 2015. Inner vs. outer A-not-A questions. Talk presented at the International Workshop on Cartography of Syntax, Beijing Language and Culture University, December 6-7.

Velleman, Dan Bridges, David Beaver, Emilie Destruel, Dylan Bumford, Edgar Onea \& Liz Coppock. 2012. It-clefts are IT (Inquiry Terminating) constructions. In Anca Chereches (ed.), Proceedings of Semantics and Linguistic Theory 22, 441-460. Ithaca, NY: CLC. doi:10.3765/salt.v22i0.2640.

Ye, Shumian. 2020a. Monopolarity and question bias: A-not-A questions in Mandarin Chinese. Talk presented at From T to C: Grammatical Representation of Tense and Speech Acts, Vietnam Institute of Linguistics, January 15-16.

Ye, Shumian. 2020b. Strategies of inquiry: Focus and contrastive topic in polar questions. Theoretical Linguistics 46(1-2). 133-139. doi:10.1515/tl-20200008.

Yuan, Mengxi \& Yurie Hara. 2015. Two ways to deploy Hamblin alternatives: Mandarin questions and unconditionals. Manuscript. https://ling.auf.net/lingbuzz/ 002767.

Shumian Ye

Department of Chinese Language and Literature

Peking University

Haidian District, Beijing, 100871, China

shumianye@gmail.com 